\title{
Erratum
}

\section{The Arecibo Galaxy Environment Survey-Potential for finding Dark Galaxies and Results so far}

\author{
R. F. Minchin, R. Auld, L. Cortese, J. I. Davies, E. Momjian, \\ R. Taylor, B. Catinella, P. Henning, S. Linder, E. Muller, \\ K. O'Neil, J. Rosenberg, S. Sabatini, S. E. Schneider, \\ M. Stage, W. van Driel, the AGES team
}

doi:10.1017/S1743921307013890, Published by Cambridge University Press 14 Feb 2008

Reproduction errors have occured in figures 1-4 of this paper, published in these proceedings, pages 112-119. The complete corrected paper is reproduced here for clarity.

Cambridge University Press apologise to the authors and readers for these errors. 


\title{
The Arecibo Galaxy Environment Survey - Potential for finding Dark Galaxies and Results so far
}

\author{
R. F. Minchin ${ }^{1}$, R. Auld ${ }^{2}$, L. Cortese ${ }^{2}$, J. I. Davies ${ }^{2}$, E. Momjian ${ }^{1}$, \\ R. Taylor ${ }^{2}$, B. Catinella ${ }^{3}$, P. Henning ${ }^{4}$, S. Linder ${ }^{5}$, E. Muller ${ }^{6}$, \\ K. O'Neil ${ }^{7}$, J. Rosenberg ${ }^{8}$, S. Sabatini ${ }^{9}$, S. E. Schneider ${ }^{10}$, M. Stage ${ }^{10}$, \\ W. van Driel $^{11}$, the AGES team \\ ${ }^{1}$ Arecibo Observatory, Arecibo, United States, email: rminchin@naic.edu, \\ ${ }^{2}$ Cardiff University, Cardiff, United Kingdom, \\ ${ }^{3}$ Max Planck Institute for Astrophysics, Garching, Germany, \\ ${ }^{4}$ University of New Mexico, Albuquerque, United States, \\ ${ }^{5}$ University of Hamburg, Hamburg, Germany, \\ ${ }^{6}$ Australia Telescope National Facility, Sydney, Australia, \\ ${ }^{7}$ National Radio Astronomy Observatory, Green Bank, United States, \\ ${ }^{8}$ Harvard Smithsonian Centre for Astrophysics, Cambridge, United States, \\ ${ }^{9}$ Osservatorio Astronomico di Roma, Rome, Italy, \\ ${ }^{10}$ University of Massachusetts, Amhurst, United States, \\ ${ }^{11}$ Observatoire de Paris, Paris, France
}

\begin{abstract}
The Arecibo Galaxy Environment Survey is a blind neutral hydrogen survey using the ALFA multibeam receiver at Arecibo Observatory to reach unprecedented sensitivities in a number of selected fields in the local Universe. When completed the survey will cover 200 square degrees out to a distance of at least $270 \mathrm{Mpc}$. If a population of gas-rich dark galaxies exists, then this survey is in a prime position to uncover that population.

So far 20 square degrees have been covered in the regions of Abell 1367, the Virgo Cluster, the NGC 7332/9 galaxy pair and the isolated galaxy NGC 1156. Over 200 sources have been found, including a number that have no obvious optical counterparts. We discuss here the potential of AGES for uncovering more such objects and the characteristics of the dark sources identified to date.
\end{abstract}

Keywords. surveys, galaxies: distances and redshifts, dark matter, radio lines: galaxies, clusters: individual (Abell 1367, Virgo), galaxies: individual (AGES J0256+2546, AGES J0300+2554, AGES J1239+1152, AGES J1245+1148, AGES J2257+2253)

\section{Introduction}

Neutral hydrogen surveys have long been proposed as a method of uncovering a population of dark galaxies that have failed to form stars (e.g. Jimenez et al. 1997, Davies et al. 2006). However, blind neutral hydrogen surveys to date have failed to turn up such a population in large numbers.

No sources that could not be identified with optical counterparts or explained in other ways (e.g. tidal debris, High Velocity Clouds, or obscured galaxies behind the plane of the Milky Way) were found in HIPASS (Doyle et al. 2005), although Davies et al. (2006) have proposed that this may be due to the difficulty in identifying the correct optical counterparts to these sources. The only good candidate dark galaxy to have been found so far in neutral hydrogen surveys is VIRGOHI 21 (Davies et al. 2004; Minchin et al. 2005; Minchin et al. 2007), although its status remains disputed (Haynes et al. 2007; Duc \& Bournaud 2007). 
Table 1. Details of the AGES regions

\begin{tabular}{lllll}
\hline Region & R.A. (J2000) & Decl. (J200) & $\begin{array}{l}\text { Extent } \\
(\text { deg.) }\end{array}$ & Structure \\
\hline AGES33PP & $01: 34: 00$ & $30: 40: 00$ & $5 \times 4$ & M33 and Pisces-Perseus \\
AGESGAL2082 & $02: 36: 00$ & $25: 48: 00$ & $2.5 \times 2$ & Isolated Galaxy UGC 2082 \\
AGESGAL1156 & $03: 00: 00$ & $25: 12: 00$ & $2.5 \times 2$ & Isolated Galaxy NGC 1156 \\
AGESGAL2577 & $08: 24: 00$ & $22: 30: 00$ & $5 \times 4$ & NGC 2577/UGC 4375 Galaxy Pair \\
AGES3193 & $10: 03: 00$ & $21: 53: 00$ & $5 \times 4$ & NGC 3193 Group \\
AGESLEO & $10: 45: 00$ & $11: 48: 00$ & $5 \times 4$ & Leo Group \\
AGES1367 & $11: 44: 00$ & $19: 50: 00$ & $5 \times 4$ & Abell 1367 Cluster \\
AGESVC1 & $12: 29: 00$ & $08: 00: 00$ & $10 \times 2$ & Virgo Cluster field 1 \\
AGESVC2 & $12: 48: 00$ & $11: 36: 00$ & $5 \times 1$ & Virgo Cluster field 2 \\
AGESGAL5523 & $14: 15: 00$ & $29: 20: 00$ & $5 \times 4$ & Isolated Galaxy NGC 5523 \\
AGESLV & $18: 38: 00$ & $18: 00: 00$ & $5 \times 4$ & The Local Void \\
AGESGAL7332 & $22: 36: 00$ & $23: 48: 00$ & $2.5 \times 2$ & NGC 7332/NGC 7339 Galaxy Pair \\
AGES7448 & $23: 00: 00$ & $15: 59: 00$ & $5 \times 4$ & NGC 7448 Group \\
\hline
\end{tabular}

The question then is - can neutral hydrogen surveys actually find dark galaxies, even if such objects exist? Davies et al. (2006) predicted that the deeper surveys now under way at Arecibo Observatory would turn up a much higher fraction of dark galaxy candidates than the earlier, relatively shallow surveys. The two large-area surveys being carried out at Arecibo are the Arecibo Legacy Fast ALFA survey (ALFALFA), which plans to cover $7000 \mathrm{deg}^{2}$ in 2 passes, and the Arecibo Galaxy Environment Survey (AGES) which will cover a smaller area of $200 \mathrm{deg}^{2}$ but to much greater sensitivity with 25 passes.

\section{The Survey}

The Arecibo Galaxy Environment Survey (AGES) is a deep, blind neutral hydrogen survey targetting thirteen fields containing different environments in the local Universe, and the effectively randomly-selected volume behind these environments. The survey began in December 2005 with observations around the isolated galaxy NGC 1156, following precursor observations in November and December 2004 (Auld et al. 2006). To date, three regions have been completed: the isolated galaxy NGC 1156, the galaxy pair NGC 7332/NGC 7339 and the Virgo Cluster field 2. A quarter of the area of the Abell 1367 region has also been observed and a start has been made on the NGC 3193 group. The regions targetted by the survey are summarised in Table 1.

The fields targetted can be divided into the following categories:

- The Local Void: The survey will cover $20 \mathrm{deg}^{2}$ in the Local Void region. If undisturbed very low surface-brightness, or even dark, galaxies are to be found, then the low-density environment of the void is a good place to look. AGES will have sufficient sensitivity over a large enough volume to place strong limits on what population may be present in this region.

- Isolated Galaxies: NGC 1156, NGC 5523 and UGC 2082 are all isolated galaxies, with no other large galaxies in their vicinity. AGES will identify whether the proportion of gas-rich dwarf companions around isolated galaxies is similar to that in groups, or lower. Identifying such galaxies optically is very challenging due to the low numbers to be found, making statistical background subtraction impossible, and the difficulty of getting optical spectra for what are often very dim objects. Neutral hydrogen provides a way of unambiguously identifying the gas-rich component of the dwarf population, and 
AGES has sufficient sensitivity to detect dwarf companions as small as $10^{7} M_{\odot}$ out to beyond $20 \mathrm{Mpc}$.

- Galaxy Pairs: NGC 7332/NGC 7339 and NGC 2577/UGC 4375 are two pairs with one large spiral and one S0 galaxy. In a similar way to around the isolated galaxies, AGES will probe the dwarf population around these systems, revealing how this is affected by different environments.

- Galaxy Groups: The NGC 7448 Group is dominated by a spiral galaxy, the NGC 3193 group is dominated by an elliptical, The Leo Group has a mix of large ellipticals and early-type spirals. Although these are similar environments in terms of their density, the different morphologies of the major galaxies indicate differing histories. AGES will discover how this has affected the population of gas-rich dwarfs and will also search for free-floating gas clouds ripped off by interactions between the galaxies in the groups.

- M33 and the Pisces-Perseus filament: With M33 in the foreground and the Pisces-Perseus filament in the background, this region will provide both a high-resolution (sub-kpc) map of a nearby galaxy and the chance to probe the high-density region of Pisces-Perseus. Observations of M33 should map its High Velocity Cloud population as well as uncovering tidal bridges and tails from its interaction with M31, or any other galaxy.

- Galaxy Clusters: $45 \mathrm{deg}^{2}$ - almost a quarter of the survey - is devoted to the Abell 1367 and Virgo clusters. Abell 1367 is a dynamically young, spiral-rich cluster with many infalling sub-clusters and opportunities to study ram-pressure stripping and tidal interactions. The Virgo Cluster, by contrast, provides a very different environment with many more elliptical galaxies and gas-poor spirals; AGES will investigate, using two separate fields, how the population changes with distance from the cluster core. The outskirts of Virgo may well be a fertile area for searching for hydrogen clouds, with objects such as HI 1225+01 (Giovanelli \& Haynes 1989) already known in the region.

- The AGES Volume: The regions of the cubes outside of the targetted structure covers a wide variety of different environments, such as the Pegasus Void in the NGC 7332 region or the WBL 091 group in the background of NGC 1156. No selection has been applied other than for the main targets, thus the AGES Volume forms a deep sample of 200 random square degrees of sky in which many interesting sources may be found.

\section{Potential for finding Dark Galaxies}

AGES reaches a sensitivity of $0.5 \mathrm{mJy}^{\text {beam }}{ }^{-1}$ to extended sources (or a columndensity of $1.6 \times 10^{17} \frac{\Delta V}{10} \mathrm{~cm}^{-2}$ ) and $0.6 \mathrm{mJy}^{-1} \mathrm{beam}^{-1}$ to point sources. Once identified on the low-noise cubes, the position and velocity can be fitted on a high-resolution cube with a beam size of $3.42 \times 3.48$ arcminutes and a velocity resolution of $6.8 \mathrm{~km} \mathrm{~s}^{-1}$. Analysis of the sources in the Abell 1367 region where optical velocities are available to confirm the identifications shows a median offset in position of 18 arcseconds (Figure 1). At the median redshift for AGES sources of $\sim 12,000 \mathrm{~km} \mathrm{~s}^{-1}$, this corresponds to $\sim 15 \mathrm{kpc}$ (for $\left.H_{0}=70 \mathrm{~km} \mathrm{~s}^{-1} \mathrm{Mpc}^{-1}\right)$. Analysis of the regions covered so far has revealed around 10 galaxies per square degree, giving a total of 2000 for the survey when completed

From the simulations of Davies et al. (2006), AGES is predicted to contain 23 per cent dark galaxies in the targetted regions. Making secure identifications between neutral hydrogen sources and their optical counterparts is a challenge for all blind H I surveys, particularly as large area surveys have very low predicted fractions (2 per cent for HIPASS, 4 per cent for ALFALFA). However, with the high percentage predicted to be dark in the AGES regions, it would seem impossible that a population as modelled by Davies et al. (2006) would be entirely lost to misidentification. Even if the population is significantly 


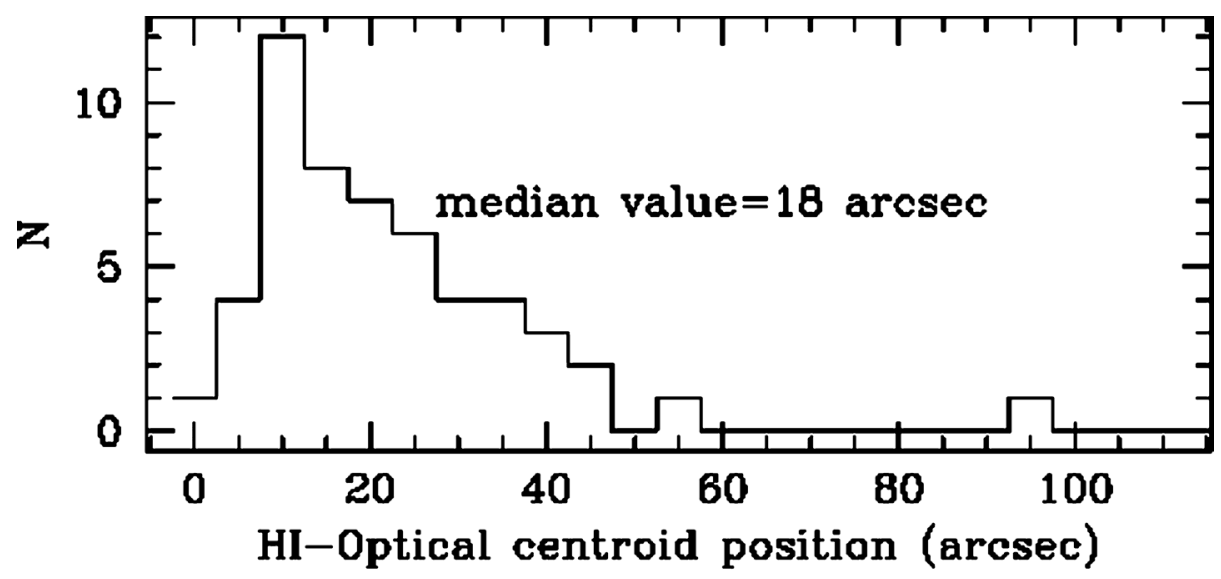

Figure 1. Offsets between neutral hydrogen sources and optical counterparts with matching optical velocities in teh Abell 1367 field.

Table 2. Properties of the dark galaxy candidates in AGES

\begin{tabular}{lllll} 
ID & Vel $\left(\mathrm{km} \mathrm{s}^{-1}\right)$ & $\Delta V_{20}\left(\mathrm{~km} \mathrm{~s}^{-1}\right)$ & $F_{H I}\left(\mathrm{Jy} \mathrm{km} \mathrm{s}^{-1}\right)$ & $M_{H I}\left(10^{8} M_{\odot}\right)$ \\
\hline AGES J0256+2546 & 10354 & 105 & 0.14 & 7.0 \\
AGES J0300+2554 & 10629 & 144 & 0.19 & 10.4 \\
AGES J1239+1152 & 6907 & 101 & 0.16 & 3.6 \\
AGES J1243+1148 & 12130 & 77 & 0.17 & 12.3 \\
AGES J2237+2253 & 11501 & 76 & 0.23 & 14.4 \\
\hline
\end{tabular}

smaller than predicted, AGES should see some evidence for its existence. Thus, even though ALFALFA is expected to find many more galaxies than AGES (Giovanelli et al. 2005), the much higher fraction predicted to be dark in AGES means that AGES should be able to place more stringent limits on any population of dark galaxies.

\section{Dark Galaxies and Lost Baryons in the survey so far}

So far, 4 regions have been at least partly analysed: NGC 1156 (Auld 2007; Auld et al. 2007, in prep.), Virgo Cluster field 2 (Taylor et al. 2007), part of Abell 1367 (Cortese et al. 2007), and NGC 7332 (Minchin et al. 2007, in prep.). These cover a total of 20 square degrees and contain around 200 sources. Five dark galaxy candidates have been identified in these fields; their properties are given in Table 2, optical images and $\mathrm{HI}$ spectra are given in Figure 2.

In addition to the dark-galaxy candidates, AGES has been turning up H I clouds without optical counterparts. Two clouds were seen at $\sim 250 \mathrm{~km} \mathrm{~s}^{-1}$ in the Virgo Cluster field (Figure 3), which have been identified as probable High Velocity Clouds (HVCs). Three clouds have also been found in the part of the Abell Cluster field that has been completed so far: two associated with the CGCG 97027 subcluster and one with the CGCG 97041 subcluster (Figure 4). All of these appear, as can be seen from the figure, to be part of extended envelopes of gas that also enclose optically-bright galaxies and are therefore likely to be interaction debris.

These H I clouds appear to be 'lost baryons' in the sense that they would not be picked up in a search based on optical targets, but are not actual dark galaxy candidates. 
(a)
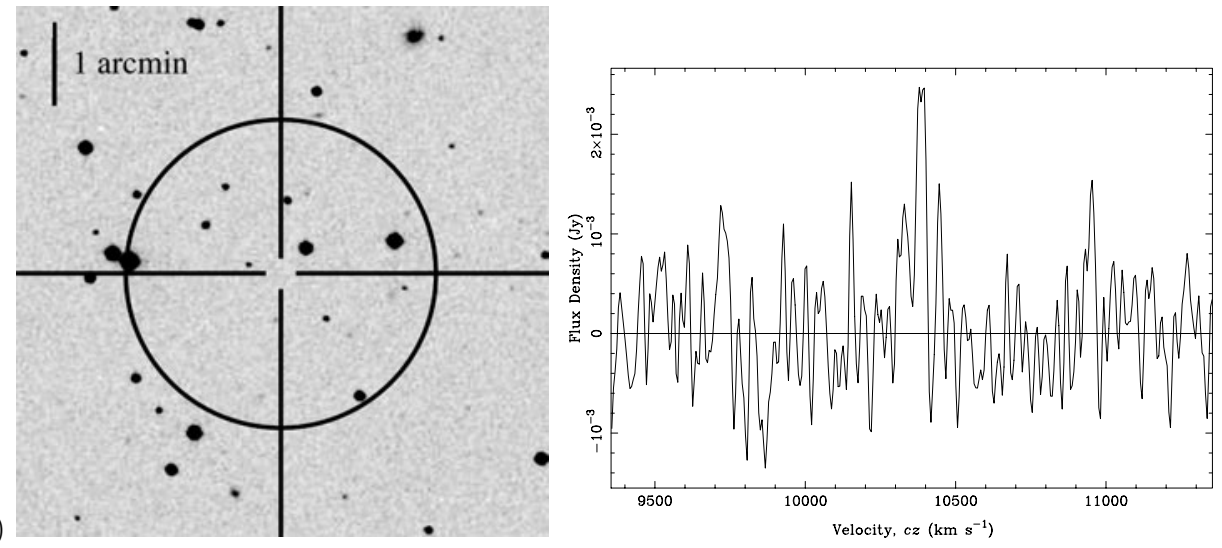

(b)
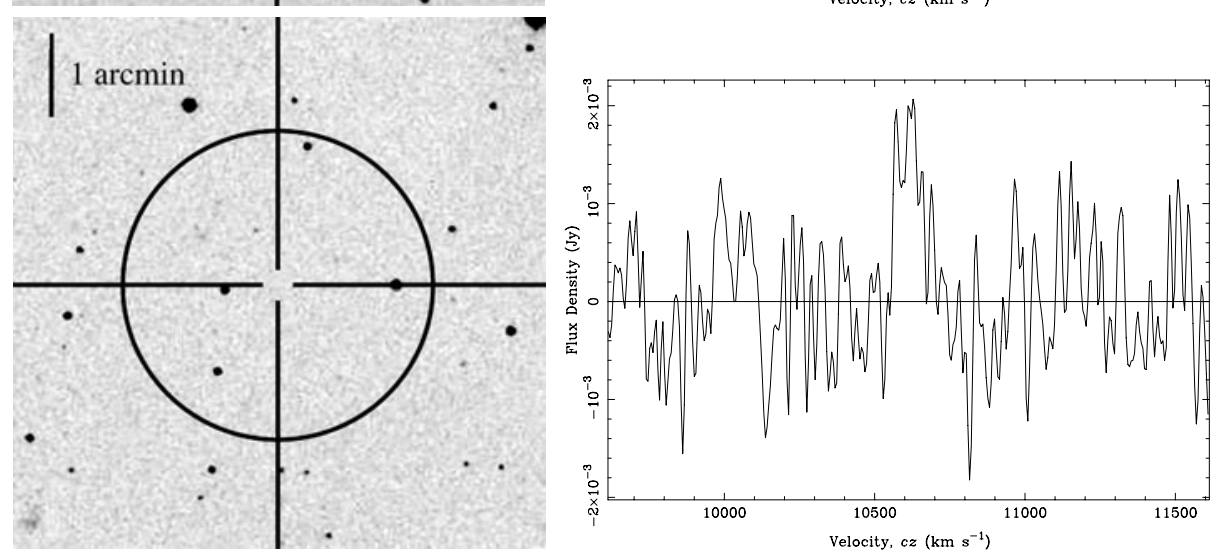

(c)
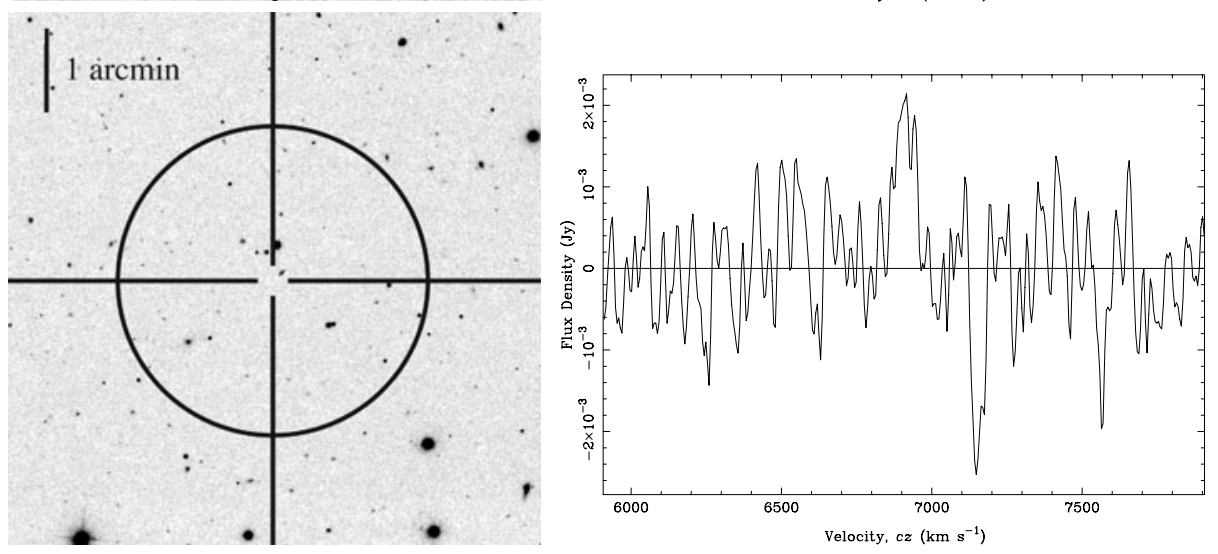

Figure 2 .

Nevertheless, they may turn out to be very interesting objects and will advance our understanding of interactions within rich environments.

\section{Discussion}

AGES has turned up six sources too faint to be found on the DSS or (for the two where it is available) the SDSS. Only one of these sources has yet been followed up with deep optical imaging, and this revealed an optical counterpart behind over a magnitude 
(d)
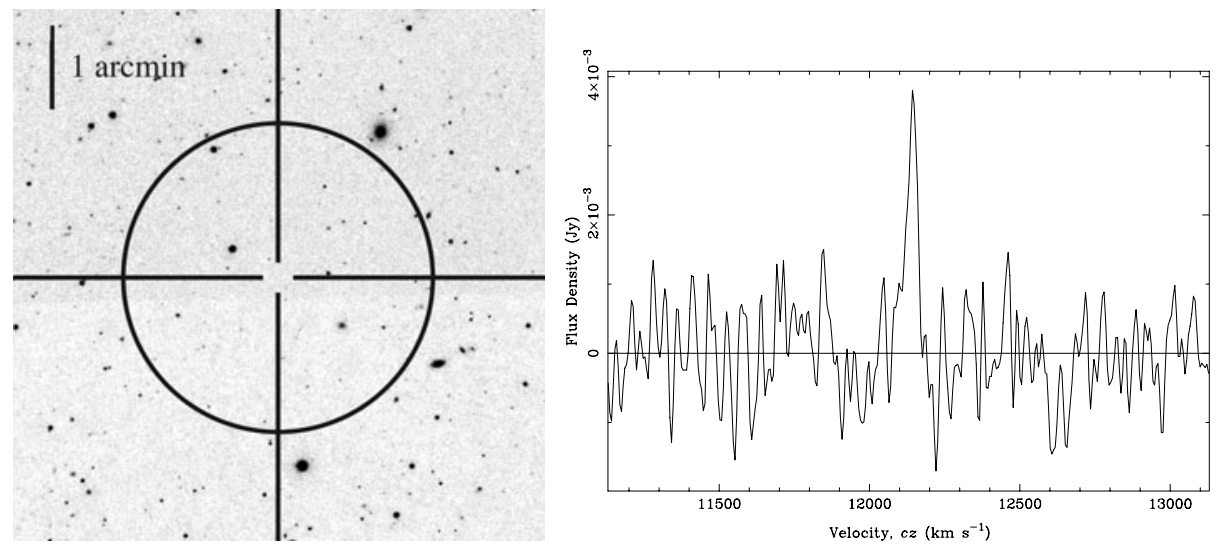

(e)
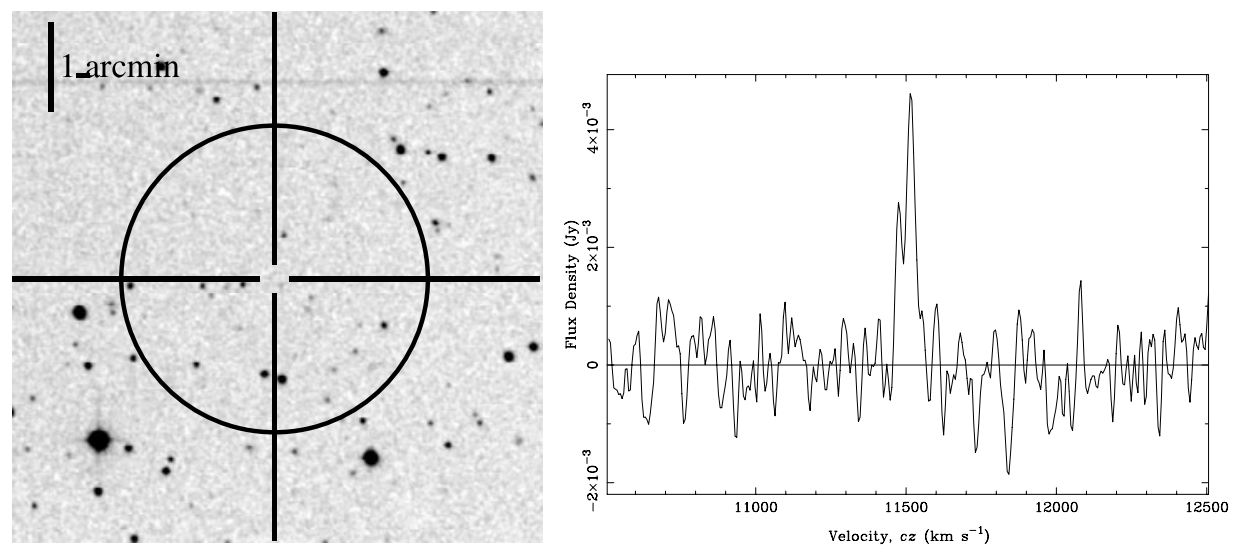

Figure 2. (continued) Potential dark galaxies in the AGES data cubes: In descending order: (a) AGES J0256+2546; (b) AGES J0300+2554; (c) AGES J1239+1152; (d) AGES J1243+1148;

(e) AGES J2237+2253. Optical images on the right are DSS2 $B$-band images for (a), (b) and (e) and SDSS images from multi-band data for (c) and (d). The circles indicate the AGES beam and the cross-hairs give the H I position. North is up and east is to the left. AGES spectra are shown on the left, Hanning smoothed to a velocity resolution of $15 \mathrm{~km} \mathrm{~s}^{-1}$.

of $B$-band extinction. The other sources lie in areas of lower extinction, but could still quite conceivably be very low surface-brightness galaxies.

All of the candidates have large neutral-hydrogen masses, much higher than VIRGOHI 21. The masses are just about within the distribution of dark galaxies modelled by Davies et al. (2006), it should be noted that if such a population exists then it would be the very highest $\mathrm{H}$ I-mass members that would be picked up most easily in a neutral hydrogen survey, in the same way that any flux-limited sample will contain mostly objects near the top end of the distribution of fluxes.

It is worth noting that all 5 of the candidates have emerged from the AGES Volume rather than from the targetted fields. This is almost certainly due to the much larger volume covered outside of the selected regions. Sufficient sources $(\sim 50$ have been found in Abell 1367 that, with 5/200 sources potential dark galaxies, one would be expected to fall in this cluster. This may be due to dark or very low surface-brightness galaxies being disrupted and/or forced into star formation on short timescales on entering the cluster, or (given the fairly small numbers involved at this stage, with only a quarter of the Abell 1367 region observed), could simply be due to small number statistics. 


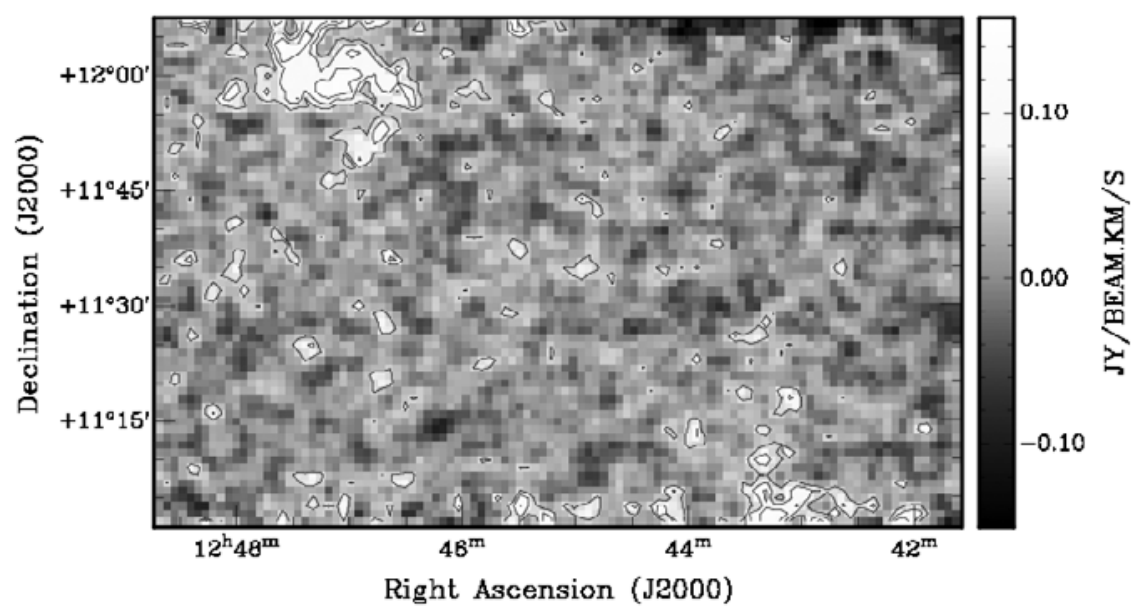

Figure 3. Neutral hydrogen clouds without optical counterpart seen at $\sim 250 \mathrm{~km} \mathrm{~s}^{-1}$ in the direction of the Virgo Cluster. These are probably local High Velocity Clouds.
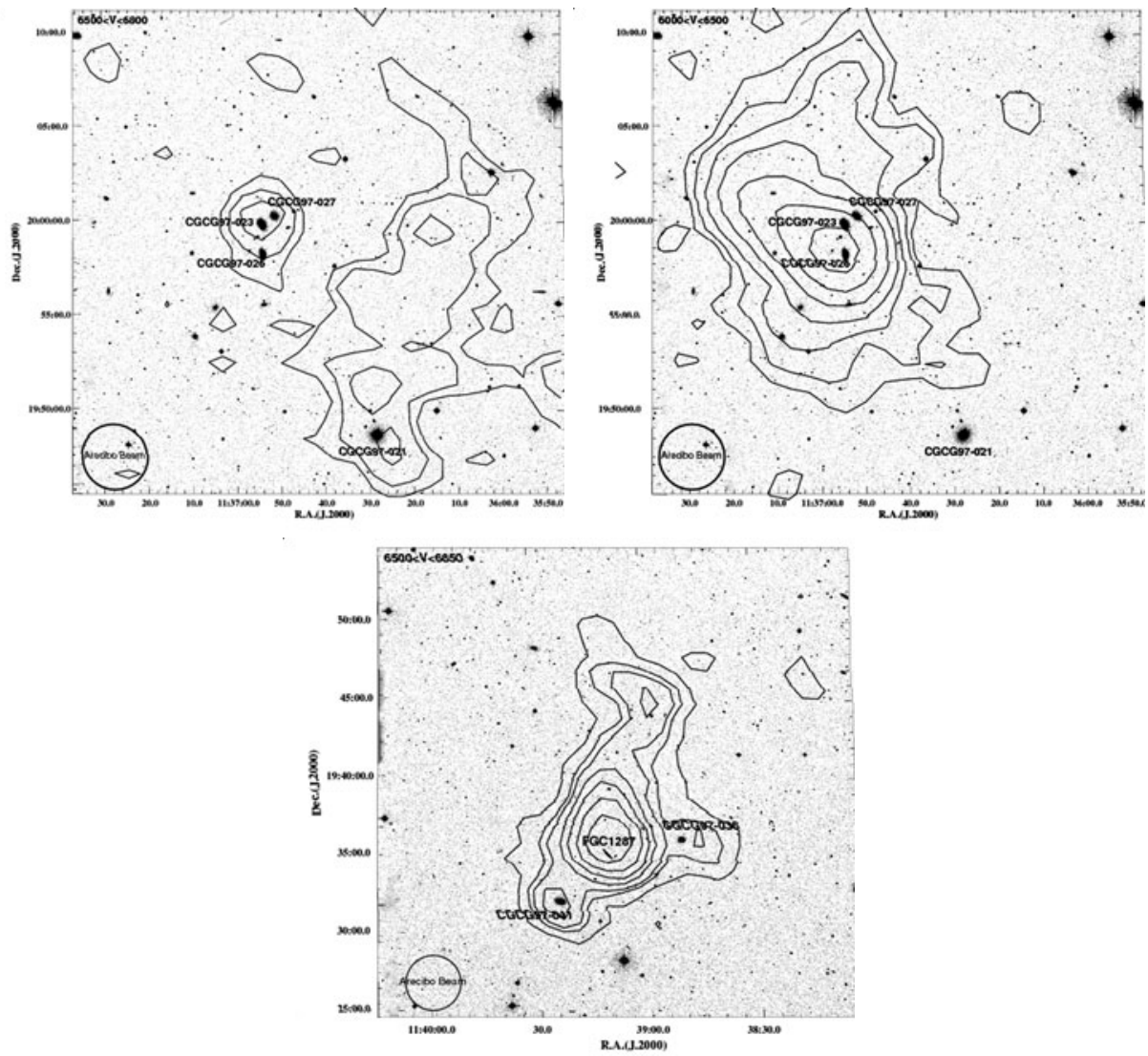

Figure 4. Extended neutral hydrogen envelopes around galaxies in the CGCG 97027 (upper) and CGCG 97041 (lower) sub-clusters of Abell 1367. 


\section{Conclusions}

AGES is the best of the current generations of neutral hydrogen surveys for uncovering a population of neutral-hydrogen rich dark galaxies. So far, with the survey 10 per cent complete, 5 candidates have been identified. This is only 2.5 per cent of the total sample of galaxies, and these still require deep optical follow-up which may identify low surfacebrightness counterparts. The evidence from AGES so far is not in favour of there being a large population of dark galaxies, but much of the survey is still to be completed so the limits are not yet very strong.

\section{Acknowledgements}

The Arecibo Observatory is part of the National Astronomy and Ionosphere Center, which is operated by Cornell University under a cooperative agreement with the National Science Foundation. This research has made use of data obtained from or software provided by the US National Virtual Observatory, which is sponsored by the National Science Foundation.

\section{References}

Auld, R. 2007, PhD Thesis, Cardiff University

Auld, R., Minchin, R. F., Davies, J. I. et al. 2006, MNRAS, 371, 1617

Cortese, L., et al. 2007, these proceedings

Davies, J. I., Disney, M. J., Minchin, R. F., Auld, R. \& Smith, R. 2006, MNRAS 368, 1479

Davies J., Minchin, R., Sabatini, S. et al. 2004, MNRAS, 349, 922

Duc, P.-A., Bournaud, F. 2007, ApJ, submitted

Giovanelli, R. et al. 2005, ApJ, 130, 2598

Giovaenlli, R. \& Haynes M. P. 1989, ApJ, 346L, 5

Jimenez, R., Heavens, A. F., Hawkins, M. R. S. \& Padoan, P. 1997, MNRAS, 292L, 5

Haynes, M. P., Giovanelli, R. \& Kent, B. R. 2007, ApJL, in press

Minchin, R., Davies, J., Disney, M. et al. 2007, ApJ, in press

Minchin, R., Davies, J., Disney, M. et al. 2005, ApJ, L21

Taylor, R., et al. 2007, these proceedings 